\title{
HARMONIC OSCILLATOR AND RELATED STATES WITH LARGE VALUE OF UNCERTAINTY
}

Vladimir A. Andreev

P.N. Lebedev Physical Institute, Russian Academy of Sciences, http://www.lebedev.ru

Moscow 119991, Russian Federation

andrvlad@yandex.ru

Abstract. A review is given of the properties of two types of quantum states, which have great uncertainty in the coordinate and momentum. Both are obtained from the states of a harmonic oscillator by means of certain transformations. The first type is correlated states, they are obtained from coherent states with a help of the Bogolyubov transform. The variances of the coordinate and momentum of such a state depend on the Bogolyubov transform parameters and can, in general, take arbitrarily large values. Their specific values are determined by the physical processes with which the Bogolyubov transform is realized. A concrete example of such physical process is considered. Another type are stretched states. Formally, they arise when the n-partial state of a harmonic oscillator undergoes a transform associated with a scale transformation of the phase space. The dispersion of the coordinate and momentum of these states depends on the scale transformation parameter and can also take arbitrarily large values. Physically stretched states can be obtained by passing n-photon states through a quantum amplifier. The role of the scale transformation of the phase space is played by the gain of the quantum amplifier.

Keywords: harmonic oscillator, correlated states, compressed states, stretched states, uncertainty relations, Planck constant

PACS 03.65.-w, 23.20.Lv

Bibliography - 33 references

Received 22.04.2017

RENSIT, 2017, 9(1):8-20

DOI: $10.17725 /$ rensit.2017.09.008

\section{Contents}

1. INTRODUCTION (8)

2. The uncertainty Relations (9)

3. The squeEzed and Correlated states (11)

4. The exitation of SQueEzed AND CORRELATED STATES (14)

5. The stretched states (16)

6. Conclusion (19)

REFerenCEs (19)

\section{INTRODUCTION}

This review aims to systematize the disparate facts related to the field of low energy nuclear reactions (LENR), as well as some unusual phenomena associated with electromagnetic field. Until recently, this area was outside of wide scientific discussion

The most of the observed phenomena of this type have no theoretical explanation, and are in contradiction with well-established ideas about the physics of processes of this kind. For this reason they were blamed for the lack of cleanliness of experiments, small statistics and low, in the opinion of critics, qualification of the authors.

In addition, some of the authors did not give the detailed description of their devices and experimental conditions, which made it impossible to verify the results independently. This raised doubts about their scrupulosity. Therefore the studies of this kind did not get support in authoritative academic community, and as the result did not get necessary financial and administrative support. They acquired an odious shade and were discarded in the marginal area.

In recent years, the situation has changed. First, the quantity turned into quality, and the abundance of results obtained in authoritative scientific centers in different countries no longer allowed to be dismissed. Secondly, and more importantly, it turned out that the development of 
this topic promises an inexhaustible, safe source of energy. And it opens the way to cheap and rapid decontamination of radioactive isotopes arising from operation of nuclear reactors.

This stimulated the activity of theoreticians in this field, which led to understanding that such phenomena and processes do not contradict the basic laws of nature and many of them can be described using traditional theoretical approaches. Here we give references to some accessible and informative sources on this topic [1-6], if desired; this list can be significantly expanded.

One of the first works in which the problem of LENR was discussed is [7].

At the same time, in our opinion, in order to explain some facts it is necessary to go beyond traditional methods and to look more broadly at the laws of nature and on the consequences that follow from them. This seems quite natural, because the range of phenomena that we discuss is very wide, and it is difficult to expect that all of them can be described in the frame of one model. In this review, we will analyze two such models: the model of correlated states and the model of stretched states.

These models are built within the framework of traditional quantum mechanics and don't use any additional physical assumptions that are different from those generally accepted. Previously they were used in other problems. In this review we describe their formal structure, but don't discuss their possible applications.

One of the phenomena that are actively discussed in connection with LENR is the tunnel effect. It is known that the probability of the occurrence of nuclear reactions involving charged particles of low or medium energies is determined, to a large extent, by the existence of a Coulomb barrier. The charged particles can pass this barrier due to the tunneling effect. The probability of the occurrence of nuclear reactions is determined by the probability of passage of charged particles through the barrier.
Therefore it is of interest to consider those factors that can increase this probability.

One of these factors is the connection between product of position and momentum dispersions with the probability of particle passing through a potential barrier. It is assumed that increase of the product of the position and momentum dispersions leads to increase of transparency of the barrier. This in turn increases the probability of nuclear reactions at low energy of the interacting particles. Generally speaking, this statement can not be considered absolutely fair, something like a mathematical theorem, sometimes it can be fulfilled, and sometimes it does not. Everything depends on the specific situation.

In this work we consider two types of states for which the uncertainties of the position and momentum can take arbitrarily large values. We didn't discuss the problem whether these states arise in atomic nucleus, and if they arise, whether they stimulate the low-energy nuclear reactions conducive.

But first we give the basic information concerning the uncertainty relations. This is necessary for unification of terminology and notation, which we will use in the future.

\section{THE UNCERTAINTY RELATIONS}

The uncertainty relations are the most important characteristic of quantum states, which distinguishes them from classical states. For quantum states one can't measure simultaneously some observable with arbitrary precision. This fact limits our possibilities in localizing this state, but on the other hand, it makes it possible to detect it outside the region of classical localization.

We give the basic facts concerning the uncertainty relations. Here we follow, basically, [8].

Let there be an observable $A$ and operator $\hat{A}$ is associated with it. By "uncertainty" of the value $A$ one understand its root-mean-square deviation, assuming 


$$
\Delta \mathrm{A}=\sqrt{\sigma_{A}} .
$$

Here $\sigma_{\mathrm{A}}$ is the dispersion of the quantity $A$

$$
\sigma_{A}=\left\langle\hat{\mathrm{A}}^{2}\right\rangle-\langle\hat{\mathrm{A}}\rangle^{2} \text {. }
$$

$$
\langle\hat{\mathrm{A}}\rangle=\int \psi^{*} \hat{\mathrm{A}} \psi d V \text {. }
$$

If the state is mixed and is described by a density matrix $\hat{\rho}$, then

$$
\langle\hat{\mathrm{A}}\rangle=S p(\hat{\rho} \hat{\mathrm{A}}) \text {. }
$$

The Heisenberg uncertainty relation was derived in 1927 for position and momentum operators. It reads [9]

$$
\sigma_{p} \sigma_{x} \geq \frac{\hbar^{2}}{4} \text {. }
$$

In 1930, independently, Robertson and Schrödinger derived an inequality, which is valid for arbitrary Hermitian operators and $[10,11]$.

$$
\sigma_{A} \sigma_{B}-\sigma_{A B}^{2} \geq \frac{\hbar^{2}}{4}|/[\hat{\mathrm{A}}, \hat{B}]\rangle \mid \text {. }
$$

Here

$$
\sigma_{A B}=\frac{1}{2}\langle\hat{\mathrm{A}} \hat{B}+\hat{B} \hat{\mathrm{A}}\rangle-\langle\hat{\mathrm{A}}\rangle\langle\hat{B}\rangle \text {. }
$$

The inequality (6) is called the RobertsonSchrödinger inequality. For the position and momentum operators it reads

$$
\sigma_{p} \sigma_{x}-\sigma_{p x}^{2} \geq \frac{\hbar^{2}}{4} \text {. }
$$

The inequalities (5) and (6) are valid for any quantum states, but in a number of cases they can be clarified. Namely, by considering certain classes of states, or even single state, one can find out that for these states the left-hand sides of the inequalities exceed the minimum values of uncertainties. First of all, we note that the inequalities (5), (6) can be transformed into equalities for pure states. If, on the other hand, the state is mixed and is described by a density matrix $\hat{\rho}$, then the inequalities (5), (6) take the form

$$
\begin{aligned}
& \sigma_{p} \sigma_{x} \geq \frac{\hbar^{2}}{4} \Phi(\mu), \\
& \sigma_{A} \sigma_{B}-\sigma_{A B}^{2} \geq \frac{\hbar^{2}}{4}|\langle[\hat{\mathrm{A}}, \hat{B}]\rangle| \Phi(\mu) .
\end{aligned}
$$

Here $\mu$ - is a purity

$$
\mu=\operatorname{Sp}\left(\hat{\rho}^{2}\right), 0<\mu \leq 1 .
$$

The quantity $\Phi(\mu)$ is a function that grows monotonically with decreasing of the parameter $\mu$, such that $\Phi(1)=1$. For pure states $\rho^{2}=\rho$ and $\mu=1$.

However even for pure states the left-hand side of the uncertainty relations may be greater than the minimum value. As an example, consider the $N$-particle states of a harmonic oscillator $|N\rangle$. For such states the relation (5) reads

$\sigma_{p} \sigma_{x}=(2 N+1) \frac{\hbar^{2}}{4}$.

And for some other more complicated states one can obtain estimates for corresponding uncertainty relations.

So, in the case of correlated states, the right-hand side of the inequalities (5), (6) is multiplied by a value $1 /\left(1-r^{2}\right)$, where $0 \leq r<1$ is the correlation coefficient. And in the case of the stretched states the right-hand side of the inequalities (5), (6) is multiplied by a value $\lambda^{-2}$, where $0<\lambda \leq 1$ is the scale transformation coefficient in the phase space. We will discuss below these states in detail. Now we note only that in all these cases one can introduce a value $\hbar_{\text {eff }}$ - the effective Planck constant.

$$
\hbar_{\text {eff }}=\hbar \sqrt{\Phi(\mu)}, \hbar_{\text {eff }}=\frac{\hbar}{\sqrt{\left(1-r^{2}\right)}}, \hbar_{e f f}=\frac{\hbar}{\lambda} .
$$

In the context of ideology of this work an increase of the uncertainty of certain states can be associated with increase of the probability of passage of quantum states through the potential barrier. The hope that such dependence exists is due to the fact that, with the appropriate election of the purity parameter $\mu$ and the correlation $r$ and the scale transformation coefficient $\lambda$, the value of the effective Planck constant $\hbar_{\text {eff }}$ can significantly exceed the value of the usual Planck constant h.

This indicates that fluctuations of position and momentum of a corresponding quantum state increase, and with the help of these fluctuations it can jump over a potential barrier. In addition, if we examine the formula for tunnel transition probability, we can see that the larger 
Planck constant, the greater the probability of a tunnel transition.

However some examples show that there is no direct connection between the uncertainty and the probability of passing through a potential barrier. So, in particular, if one has a wave function $\psi=c_{1} \psi_{1}+c_{2} \psi_{2}$, that is a superposition of two wave functions whose amplitude maxima are located on the axis $X$ on one side of the potential barrier, then the transmission $T$ through a potential barrier for such a function reads

$$
T=\left|c_{1}\right|^{2} T_{1}+\left|c_{2}\right|^{2} T_{2},
$$

here $T_{1}$ and $T_{2}$ are the transmission coefficients, which correspond separately to wave functions $\psi_{1}$ and $\psi_{2}$.

The expression (13) does not depend on localization of the wave packets in relation to each other, but the uncertainty relation for the function $\psi$ depends on it. By expanding the locations of localization of the wave packets relative to each other, one can increase the uncertainty of the wave function, but the transmission coefficient (13) will not change. It is the exact result, and it shows that in each particular case the relationship between the uncertainty of the wave function and the probability of passing through the potential barrier must be studied separately.

This problem was studied also in $[12,13]$. In Ref. [12] the evolution of Gaussian wave packet was studied in presence of the repulsive deltapotential barrier. For the initial packet localized far enough from the barrier, the transmission coefficient was determined as the probability of detecting a particle on the whole semi axis on the other side of the barrier. This coefficient depends on two dimensionless parameters: the normalized ratio of the force of the potential to the initial average value of the pulse and the ratio of the initial dispersion of the pulse to the initial average value of the pulse.

It was shown that for small values of the second parameter the result reduces to the known transparency formula for the delta barrier obtained in the plane wave approximation by solving the stationary Schrödinger equation. For large values of the second parameter the transmission coefficient can be much larger than that calculated in the plane-wave approximation.

In Ref. [13] the problem of tunneling a wave packet through Coulomb barrier was considered. It was shown that the value of the transmission coefficient can differ greatly from the standard expression obtained in the plane wave approximation (WKB), and can exceed it many times. It is established that the value of the transmission coefficient depends strongly on the shape of the packet.

We also note the paper [14], in which the relationship between the Heisenberg uncertainty relation and the measurement-perturbation error relation was studied.

In fact, in addition to the above, there are many other states with a large uncertainty. Our interest in correlated and stretched states is due to the fact that the processes with which they can be generated are known.

So, in particular, about 20-30 years ago many scientific groups, both in Russia and abroad, studied the squeezed light and developed ways of generating it. But they interested only in squeezing ratio, and did not notice the correlation coefficient. It seems to us interesting to analyze now these works on the subject of what correlation coefficients arise in their states.

\section{THE SQUEEZED AND CORRELATED STATES}

One can try to explain LENR with the help of squeezed and correlated states. From a formal point of view these states are special generalizations of the coherent states of harmonic oscillator. The important fact that for these states, the dispersions of position and momentum can take different values, depending on the parameters of the states. These values can be arbitrarily large, which makes it possible 
to connect these states to phenomena of an increase of the probability of passage of particles through a potential barrier.

The real and imaginary parts of the electromagnetic field in a coherent state $|\alpha\rangle$ undergo fluctuations with equal dispersions. The ground state of a harmonic oscillator is a special case of a coherent state with $\alpha=0$. For these states, the region of distribution of the canonical variables in the phase space has the form of a circle. The wave packet of the coherent state moves in the oscillator potential between the classical turning points, preserving its shape. The width of this wave packet is identical to the width of the wave packet of the ground state of the oscillator.

There exist states in which distribution of the canonical variables in the phase space is deformed in such a way that dispersion of one canonical variable decreases due to increase of dispersion of the other canonical variable. The domain of distribution of the canonical variables takes an oval shape, similar to an ellipse. Such deformation of a distribution region is called "squeezing", and such states themselves are called the "squeezed states." In contrast to coherent states, the width of the wave packet of squeezed states oscillates when these packets move back and forth in the oscillator potential.

The squeezed states were actively investigated, both theoretically and experimentally. Various methods for their generation have been proposed and realized. However, the fact that they are often also the correlated states remained in the shadows. Of course, this fact was noted by some authors, but it was not used in any way. At present, the presence of internal correlations in such states attracts attention to them. It is this fact that is used in [15-17] to explain the LENR.

In this section, we describe the main properties of squeezed and correlated states, as well as some methods for their generation.
Let us give a formal definition of the squeezed and correlated states, and describe their main properties [8,18]. As was already noted, these states are a generalization of coherent state. Let's consider a one-dimensional harmonic oscillator. Its Hamiltonian has the form

$$
\hat{H}=-\frac{\hbar^{2}}{2 m} \frac{d^{2}}{d x^{2}}+\frac{m \omega^{2}}{2} x^{2}=\frac{1}{2 m}\left(\hat{p}^{2}+m^{2} \omega^{2} \hat{q}^{2}\right)=\hbar \omega\left(\hat{a}^{\dagger} \hat{a}+\frac{1}{2}\right),
$$

The position and momentum operators $\hat{q}$ and $\hat{p}$, as well as the creation and annihilation operators, have the form

$$
\begin{aligned}
& \hat{p}=-i \hbar \frac{d}{d x}, \hat{q}=x, \\
& \hat{a}^{\dagger}=\sqrt{\frac{m \omega}{2 \hbar}} \hat{q}-i \sqrt{\frac{1}{2 m \omega \hbar}} \hat{p}, \hat{a}=\sqrt{\frac{m \omega}{2 \hbar}} \hat{q}+i \sqrt{\frac{1}{2 m \omega \hbar}} \hat{p} .
\end{aligned}
$$

The operators (15), (16) satisfy the commutation relations

$[\hat{p}, \hat{q}]=-i \hbar,\left[\hat{a}, \hat{a}^{\dagger}\right]=1$.

The position and momentum operators are connected with creation-annihilation operators as follows

$\hat{q}=\sqrt{\frac{\hbar}{2 m \omega}}\left(\hat{a}^{\dagger}+\hat{a}\right), \hat{p}=i \sqrt{\frac{m \omega \hbar}{2}}\left(\hat{a}^{\dagger}-\hat{a}\right)$.

The $n$ - partial states $|n\rangle$ of the harmonic oscillator are eigenfunctions of the Hamiltonian (14)

$$
\hat{H}|n\rangle=\hbar \omega\left(n+\frac{1}{2}\right)|n\rangle \text {. }
$$

The coherent states of a harmonic oscillator read

$$
|\alpha\rangle=e^{-|\alpha|^{2} / 2} \sum_{n=0}^{\infty} \frac{\alpha^{n}}{\sqrt{n !}}|n\rangle .
$$

Here $\alpha$ - is an arbitrary complex number.

The states (20) satisfy the equation

$$
\hat{a}|\alpha\rangle=\alpha|\alpha\rangle \text {. }
$$

The relation (21) can be regarded as the definition of a coherent state.

The coherent state $|\alpha\rangle$ can be obtained from the vacuum state $|0\rangle$ with the help of the shift operator $\hat{D}(\alpha)=\exp \left(\alpha \hat{a}^{\dagger}-\alpha^{*} \hat{a}\right)$.

$$
|\alpha\rangle=\hat{D}(\alpha)|0\rangle \text {. }
$$


Using the definition (18) of the operators $\hat{q}, \hat{p}$, one can find their mean values in the coherent state (20).

$$
\langle\alpha|\hat{q}| \alpha\rangle=\sqrt{\frac{2 \hbar}{m \omega}} \operatorname{Re} \alpha,\langle\alpha|\hat{p}| \alpha\rangle=\sqrt{2 \hbar m \omega} \operatorname{Im} \alpha .
$$

One can also calculate the dispersions of operator $\hat{q}, \hat{p}$.

$$
\begin{aligned}
& \sigma_{q}=\left\langle\alpha\left|\hat{q}^{2}\right| \alpha\right\rangle-\langle\alpha|\hat{q}| \alpha\rangle^{2}=\frac{\hbar}{2 m \omega}, \\
& \sigma_{p}=\left\langle\alpha\left|\hat{p}^{2}\right| \alpha\right\rangle-\langle\alpha|\hat{p}| \alpha\rangle^{2}=\frac{1}{2} m \omega \hbar .
\end{aligned}
$$

It follows from (24) that

$$
\sigma_{q} \sigma_{p}=\frac{1}{4} \hbar^{2}
$$

Thus, we see that for the coherent states (20) the Heisenberg uncertainty relation takes the form of equality, i.e. these states have minimal uncertainty.

Let's consider a linear transformation of creation-annihilation operators.

$$
\hat{b}=u \hat{a}+v \hat{a}^{\dagger}, \hat{b}^{\dagger}=v^{*} \hat{a}+u^{*} \hat{a}^{\dagger} .
$$

In the case when the complex numbers $u, v$ satisfy the relation

$$
|\mathrm{u}|^{2}-|\mathrm{v}|^{2}=1
$$

the operators $\hat{b}^{\dagger}, \hat{b}$ satisfy the commutation relation

$$
\left[\hat{b}, \hat{b}^{\dagger}\right]=1 \text {. }
$$

Such transform is called the Bogolyubov transform.

In this case the operators $\hat{b}^{\dagger}, \hat{b}$ can be considered as some new creation-annihilation operators. For these operators one can introduce a vacuum state $|0\rangle_{\beta}$ and a coherent state $|\beta\rangle$. These states are determined by the relations

$$
\hat{b}|0\rangle_{\beta}=0, \hat{b}|\beta\rangle=\beta|\beta\rangle,\langle\beta \mid \beta\rangle=1 \text {. }
$$

The coherent state $|\beta\rangle$ can be obtained from the vacuum state $|0\rangle_{\beta}$ in a way similar to (22)

$$
|\beta\rangle_{\beta}=\hat{D}(\beta)|0\rangle_{\beta} \text {. }
$$

The shift operator reads $\hat{D}(\beta)=\exp \left(\beta \hat{b}^{\dagger}-\beta^{*} \hat{b}\right)$. In the coordinate representation the state $|\beta\rangle$ reads

$$
\psi_{\beta}(x)=\left[\frac{\omega(|u|+|v|)^{2}}{\pi \hbar}\right]^{1 / 4} \exp \left\{-\frac{\omega}{2 \hbar} \frac{u-v}{u+v}\left[x-\left(\frac{2 \hbar}{\omega}\right)^{1 / 2} \frac{\beta}{u+v}\right]^{2}\right\} .
$$

When $u=1, v=0$ the expression (31) is the wave function of an ordinary coherent state.

The transformation (26) is given by two complex numbers $u=|u| e^{i \varphi_{u}}$ and $v=|v| e^{i \varphi_{v}}$, which satisfy the relation (27). Let's introduce the following notation

$$
\varphi=\varphi_{u}, \quad \theta=-\varphi_{u}-\varphi_{v}, \quad \tau=\ln (|u|+|v|) .
$$

It follows from (27) that $\tau \geq 0$.

The mean values of the operators $\hat{q}, \hat{p}$, given by (18), for the coherent state (30) have the form.

$$
\begin{aligned}
& \langle\beta|\hat{q}| \beta\rangle=\sqrt{\frac{2 \hbar}{m \omega}}\left[\left(u^{*}-v^{*}\right) \beta+(u-v) \beta^{*}\right], \\
& \langle\beta|\hat{p}| \beta\rangle=-i \sqrt{2 \hbar m \omega}\left[\left(u^{*}-v^{*}\right) \beta-(u-v) \beta^{*}\right] .
\end{aligned}
$$

The dispersions of the operators $\hat{q}, \hat{p}$ for the coherent state $|\beta\rangle$ have the form.

$$
\begin{aligned}
& \sigma_{q}(\beta)=\frac{\hbar}{2 m \omega}[\operatorname{ch}(2 \tau)-\operatorname{sh}(2 \tau) \cos (2 \varphi+\theta)], \\
& \sigma_{p}(\beta)=\frac{m \omega \hbar}{2}[\operatorname{ch}(2 \tau)+\operatorname{sh}(2 \tau) \cos (2 \varphi+\theta)] .
\end{aligned}
$$

The Heisenberg uncertainty relation for the state $|\beta\rangle$ reads

$$
\begin{aligned}
& \sigma_{q}(\beta) \sigma_{p}(\beta)=\frac{\hbar^{2}}{4}\left[\operatorname{ch}^{2}(2 \tau)-\operatorname{sh}^{2}(2 \tau) \cos ^{2}(2 \varphi+\theta)\right]= \\
& =\frac{\hbar^{2}}{4}\left[1+\operatorname{sh}^{2}(2 \tau) \sin ^{2}(2 \varphi+\theta)\right] \geq \frac{\hbar^{2}}{4} .
\end{aligned}
$$

One can see from formulas (34), (35) for the state (30) that the values of dispersions of position and momentum are changed, and the total value of the uncertainty is changed too. It is the result of transformation (26). In this case the dispersions can both increase and decrease depending on the values of the transformation parameters (32), but their product always increases, i.e. the uncertainty of the transformed coherent state (30) increases.

It is convenient to characterized the properties of such states $|\beta\rangle$ by two parameters: a correlation coefficient 


$$
\begin{aligned}
& r=\frac{\operatorname{Im}\left(u v^{*}\right)}{\sqrt{\left(1 / 2+|v|^{2}\right)-\left(\operatorname{Re}\left(u v^{*}\right)\right)^{2}}}= \\
& =\frac{\sin (\theta+2 \varphi)}{\sqrt{c t h^{2} 2 \tau-\cos ^{2}(\theta+2 \varphi)}},
\end{aligned}
$$

and a squeezing ratio $k$

$$
\begin{aligned}
& k=\frac{1 / 2+|v|^{2}-\left(\operatorname{Re}\left(u v^{*}\right)\right)^{2}}{1 / 2+|v|^{2}+\left(\operatorname{Re}\left(u v^{*}\right)\right)^{2}}= \\
& {\left[1-\frac{\cos (\theta+2 \varphi)}{\operatorname{cth} 2 \tau}\right]\left[1+\frac{\cos (\theta+2 \varphi)}{\operatorname{cth} 2 \tau}\right]^{-1} .}
\end{aligned}
$$

With the help of the expressions (36) and (37) one can express the product of the dispersions through the correlation coefficient

$$
\sigma_{q}(\beta) \sigma_{p}(\beta)=\frac{\hbar^{2}}{4\left(1-r^{2}\right)} \text {. }
$$

We see that the product of the dispersions that enters into the Heisenberg uncertainty relation depends on the correlation coefficient, but does not depend on the sqeezing ratio. By choosing the parameters (32) of the transformation (26) in the required manner one can obtain different values of correlation and squeezing coefficients.

So, for example, if $\theta+2 \varphi=0$, we have $r=0, k=e^{-4 \tau}$.

In this case the squeezed states are appeared, but they are uncorrelated. The values of the dispersion (34) read

$$
\sigma_{q}(\beta)=\frac{\hbar}{2 m \omega} e^{-2 \tau}, \sigma_{p}(\beta)=\frac{m \omega \hbar}{2} e^{2 \tau} .
$$

We see that the value of the dispersion of position decreases and the value of the dispersion of momentum increases. If $\theta+2 \varphi=\pi / 2$ then

$$
\sigma_{q}(\beta)=\frac{\hbar}{2 m \omega} e^{2 \tau}, \sigma_{p}(\beta)=\frac{m \omega \hbar}{2} e^{-2 \tau} .
$$

In this case on the contrary, the dispersion of position increases, and the dispersion of momentum decreases. But in both cases (40) and (41) the Heisenberg uncertainty relation has the form (25).

If $\theta+2 \varphi=\pi / 2$, then $r=\operatorname{th} 2 \tau, k=1$.
Such states do not have squeezing, but there exist correlation. The dispersions (34) read

$\sigma_{q}(\beta)=\frac{\hbar}{2 m \omega} \operatorname{ch}(2 \tau), \sigma_{p}(\beta)=\frac{m \omega \hbar}{2} \operatorname{ch}(2 \tau)$.

The product of the dispersions (43) has the form

$$
\sigma_{q}(\beta) \sigma_{p}(\beta)=\frac{\hbar^{2}}{4} c h^{2} 2 \tau \text {. }
$$

We see that for such states the region of distribution of the canonical variables in the phase space preserves the symmetric form of the circle, but the radius of this circle increases.

Let us now consider some methods of generating squeezed and correlated states.

\section{THE EXITATION OF SQUEEZED AND CORRELATED STATES}

From the formal point of view the simplest way to excite squeezed and correlated states is a parametric excitation of a harmonic oscillator. This is due to the fact that one can find exact solutions of the nonstationary Schrödinger equation for an oscillator with a variable frequency and analyze their properties.

Let's consider a nonstationary Schrödinger equation

$$
i \hbar \frac{\partial \psi(x, t)}{\partial t}=\frac{1}{2}\left[-\frac{\partial^{2}}{\partial x^{2}}+\omega^{2}(t) x^{2}\right] \psi(x, t) .
$$

Its solution has a form

$$
\begin{aligned}
& \psi_{\beta}(x, t)=\left[\frac{\omega_{0}}{\pi \hbar \epsilon^{2}(t)}\right]^{1 / 4} \times \\
& \times \exp \left[\frac{i \dot{\epsilon}(t)}{2 \hbar \epsilon(t)} x^{2}+\left(\frac{2 \omega_{0}}{\hbar}\right)^{1 / 2} \frac{\beta}{\epsilon(t)} x-\frac{\beta^{2} \epsilon^{*}(t)}{2 \epsilon(t)}-\frac{|\beta|^{2}}{2}\right] .
\end{aligned}
$$

The solution (46) depends on the function $\epsilon(t)$ , which is a solution of the equation

$$
\frac{d^{2} \epsilon(t)}{d t^{2}}+\omega^{2}(t) \epsilon(t)=0
$$

with the initial condition

$$
\epsilon(0)=1, \dot{\epsilon}(0)=i \omega_{0} \text {. }
$$

The solutions of equation (47) can not be written in an explicit form, but their properties are well investigated. If $\omega(t)$ is a periodic 
function the equation (47) is reduced to the Mathieu and Hill equations.

We introduce the parameters

$$
u=\frac{1}{2}\left(\frac{\dot{\epsilon}(t)}{i \omega_{0}}+\epsilon(t)\right), v=\frac{1}{2}\left(\frac{\dot{\epsilon}(t)}{i \omega_{0}}-\epsilon(t)\right)
$$

The quantities (49) satisfy the relation (27). Now the function (46) takes the form (31). With their help one can also find the correlation coefficient (36) and the squeezing ratio (37).

In practice, one can generate squeezed states with the help of nonlinear processes. As an example consider the degenerate parametric down-conversion process.

In this process, the light beam with a frequency $\omega_{0}$ falls on a nonlinear crystal, and as a result of interaction, the photon with frequency $\omega_{0}$ decays into two photons with frequency $\omega_{1}=\frac{1}{2} \omega_{0}$. It is assumed that the incident pump field is sufficiently intense to be considered as classical, but the produced photons must be considered in a quantum fashion. Under such assumptions, the interaction Hamiltonian reads [19]

$$
\hat{H}=\hbar \hat{a}_{1}^{\dagger} \hat{a}_{1}+\hbar g\left(\hat{a}_{1}^{\dagger 2} v_{0} e^{-2 i \omega_{1} t}+v_{0}^{*} \hat{a}_{1}^{2} e^{2 i \omega_{1} t}\right) \text {. }
$$

Here $v_{0}$ - is the complex amplitude of the initial light beam, and $g$ - is the real coupling constant, its value depends on the nonlinear susceptibility of the medium. The Heisenberg equation of motion for the operator $\hat{a}(t)$ reads

$$
\frac{d}{d t} \hat{a}_{1}=\frac{1}{i \hbar}\left[\hat{a}_{1}, \hat{H}\right]=-i \omega_{1} \hat{a}_{1}-2 i g \hat{a}_{1}^{\dagger 2} v_{0} e^{-2 i \omega_{1} t} .
$$

The general solution of the equation (51) has the form

$$
\begin{aligned}
& \hat{a}_{1}(t)=\hat{a}_{1}(0) \operatorname{ch}\left(2 g\left|v_{0}\right| t\right) e^{-i \omega_{1} t}- \\
& -i \frac{v_{0}}{\left|v_{0}\right|} \hat{a}_{1}^{\dagger}(0) \operatorname{sh}\left(2 g\left|v_{0}\right| t\right) e^{-i \omega_{1} t}, \\
& \hat{a}_{1}^{\dagger}(t)=\hat{a}_{1}^{\dagger}(0) \operatorname{ch}\left(2 g\left|v_{0}\right| t\right) e^{i \omega_{1} t}+ \\
& +i \frac{v_{0}^{*}}{\left|v_{0}\right|} \hat{a}_{1}(0) \operatorname{sh}\left(2 g\left|v_{0}\right| t\right) e^{i \omega_{1} t} .
\end{aligned}
$$

Comparing the expressions (26) and (52) one can see that solutions (52) have the form of Bogolyubov transform also. Therefore, in the process of a degenerate parametric down conversion, the creationannihilation operators $\hat{a}_{1}^{\dagger}(t), \hat{a}_{1}(t)$ of the generated photons evolve in such a way that a vacuum state $|0\rangle_{1}$, such that $\left|\hat{a}_{1}(0)\right\rangle_{1}=0$, is transformed into a squeezed state. The other examples of non-linear processes, including non-degenerate parametric down conversion, which can also be used to generate squeezed states, can be found in the book [20].

Let us find now the values of the dispersions of the position and momentum (34) and the values of correlation and squeezing coefficients (36), (37) for the coherent state (30) corresponding to the creation-annihilation operators $\hat{a}_{1}^{\dagger}(t), \hat{a}_{1}(t)$ given by formulas (52).

In this case the quantities defining the Bogolyubov transform (26) have the form

$$
\begin{aligned}
& u=\operatorname{ch}\left(2 g\left|d_{0}\right| t\right) e^{-i \omega_{1} t}, \\
& v=-i e^{i \delta} \operatorname{sh}\left(2 g\left|d_{0}\right| t\right) e^{-i \omega_{1} t} ; d_{0}=\left|d_{0}\right| e^{i \delta} .
\end{aligned}
$$

The parameters (32) read

$$
\begin{aligned}
& \varphi=\varphi_{u}=-\omega_{1} t, \theta=-\varphi_{u}-\varphi_{v}=-\delta+\pi / 2, \\
& \tau=\ln (|u|+|\nu|)=2 g\left|d_{0}\right| t .
\end{aligned}
$$

The dispersions (34) are equal to

$$
\begin{aligned}
& \sigma_{q}(\beta)=\frac{\hbar}{2 m \omega}\left[\operatorname{ch}\left(4 g\left|d_{0}\right| t\right)-\operatorname{sh}\left(4 g\left|d_{0}\right| t\right) \sin \delta\right], \\
& \sigma_{p}(\beta)=\frac{m \omega \hbar}{2}\left[\operatorname{ch}\left(4 g\left|d_{0}\right| t\right)+\operatorname{sh}\left(4 g\left|d_{0}\right| t\right) \sin \delta\right] .
\end{aligned}
$$

And the correlation and squeezing coefficients take the form

$$
\begin{aligned}
& r=\frac{\cos \delta}{\sqrt{\operatorname{cth}^{2}\left(4 g\left|d_{0}\right| t\right)-\sin ^{2} \delta}}, \\
& k=\frac{\operatorname{cth}\left(4 g\left|d_{0}\right| t\right)-\sin \delta}{\operatorname{cth}\left(4 g\left|d_{0}\right| t\right)+\sin \delta} .
\end{aligned}
$$

One can see from (55) - (57) that as time $t$ increases, both correlation and squeezing coefficients tend to unity $(r, k \rightarrow 1)$. Thus 
in the given process, as the time increases, the correlation increases, dispersions and uncertainty grow, but squeezing decreases. The domain of distribution of canonical variables expands and becomes more and more symmetric.

We have considered the simplest scheme for generating squeezed and correlated states, but there are exist other nonlinear optical processes that also lead to appearance of such states [21]. For example, if a nonlinear medium is inserted into an optical cavity, then the process of generating sqeezed and correlated states will proceed more intensively. Four-wave mixing can also be used as a nonlinear process.

\section{THE STRETCHED STATES}

In this section we consider a new approach to constructing quantum states that is arise in certain physical processes.

It was developed within the framework of cooperation between FIAN and the Serbian Academy of Sciences and Art. The main ideas of the method and the results obtained with its help are presented in Ref. [22-27].

The method is based on the quasiprobability distributions, which are used for description of quantum states. These distributions are defined at the phase space, and transformations of this space are compared to some physical processes. The transformations of the phase space induce transformations of functions that are defined at it. It is possible to find physical states that correspond to the transformed quasi-probability distributions, and after that one can find the result of the action of the physical process at the initial quantum state.

This is the general outline of our approach. In this paper the Husimi function is used as quasi-probability distribution, and amplification of quantum states is considered as a physical process. This process can be connected with a scale transformation of the phase space.

In the framework of this approach we consider the class of states for which the values of position and momentum dispersions depend on parameters of the processes by which these states are generated. In this respect they are similar to the correlated states. We will consider the states that arise when $N$-partial Fock states are subjected by the action of a quantum amplifier. We call such states - the stretched states.

The stretched states are arise as a result of scale transformation of the phase space

$(q, p) \rightarrow(\lambda q, \lambda p) ;|\lambda|^{2} \leq 1$

It was proved in $[22,23]$ that if $Q(q, p)$ is a Hushimi function of a quantum state and $\lambda<1$, then the quantity

$Q_{\lambda}(q, p)=\lambda^{2} Q(\lambda q, \lambda p)$

is also a Hushimi function of some quantum state.

For the states of a harmonic oscillator it is possible to find an exact result of such transformation. If we consider a pure state that is an arbitrary superposition of $n$-particle states, then as a result of this transformation it goes into a mixed state and one can find an explicit form of its density matrix.

This mixed $\lambda$-state contains an infinite set of pure states, and the probabilities with which these pure states enter into the mixed state form a negative binomial distribution. In the case when we are dealing initially with one $N$-particle state, the transformed state contains all $N, N+1, \ldots$-particle states. The distribution of these pure states in the mixed state becomes the more smooth the smaller parameter $\lambda^{2}$.

Simply speaking, one can consider that $\lambda$-transformation (58) of a state $|N\rangle$ generates states with large $M>N$. We call such mixed 
states — the "stretched states". The mean values of a particle number operator were found for them, and the von Neumann entropy was calculated. The form of the Heisenberg and Robertson-Schrödinger uncertainty relations for stretched states is also found. It is established that for such states the factor $\lambda^{-4}$ appears in the righthand side of the uncertainty relations. Therefore when the $\lambda$-transformation (58) is performed, the uncertainty of the states increases.

Let us define the Husimi function. Suppose there is a quantum state, which is determined by the density operator $\hat{\rho}$. Then with the help of coherent states (20) one can construct its Husimi function.

$$
Q\left(\alpha, \alpha^{*}\right)=\frac{1}{\pi} \int\langle\alpha \mid x\rangle \rho(x, y)\langle y \mid \alpha\rangle d x d y .
$$

If a quantum state is pure and is described by a wave function $|\psi\rangle$, then its Husimi function reads

$$
Q(q, p)=\langle\alpha \mid \psi\rangle\langle\psi \mid \alpha\rangle \text {. }
$$

The transformation (58) can be related to certain physical processes, for example, the passage of the state of the electromagnetic field through a quantum amplifier [28, 29]. The main idea can be understood by the example of a simple linear light amplifier. It consists of partially inverted two-level atoms.

The resonance interaction Hamiltonian of the field with atoms reads

$$
\hat{H}=\hbar k\left(\begin{array}{cc}
0 & \hat{a} \\
\hat{a}^{\dagger} & 0
\end{array}\right) \text {. }
$$

This is the interaction Hamiltonian of the Jaynes-Cummings model; it has a number of interesting properties, in particular, the property of supersymmetry [30,31]. With such a Hamiltonian, the equation for the density matrix of the electromagnetic field has in first approximation the form [32]

$$
\begin{aligned}
& \frac{\partial \hat{\rho}}{\partial t}=-k N_{1}\left(\hat{a} a^{\dagger} \hat{\rho}-2 \hat{a}^{\dagger} \hat{\rho} \hat{a}+\hat{\rho} \hat{a} \hat{a}^{\dagger}\right)- \\
& -k N_{2}\left(\hat{a}^{\dagger} \hat{a} \hat{\rho}-2 \hat{a} \hat{\rho} \hat{a}^{\dagger}+\hat{\rho} \hat{a}^{\dagger} \hat{a}\right) .
\end{aligned}
$$

Here $\hat{a}^{\dagger}, \hat{a}$ - are the creation-annihilation operators of the electromagnetic field, $N_{1}, N_{2}$ - the populations of upper and lower levels of two-level atoms, and $k$ - is the gain.

Using the relation (60) between the density matrix and the Husimi function, one can pass from the operator equation (63) to an ordinary differential equation for Husimi function. With the help of this equation an expression for Husimi function of a state at output from a quantum amplifier was found in Ref. [28]. It has the form

$$
Q_{o u t}(\alpha, t)=\frac{1}{G^{2}} Q_{i n}(\alpha / G)=\left\langle\frac{\alpha}{G}\left|\hat{\rho}_{i n}\right| \frac{\alpha}{G}\right\rangle,
$$

here

$$
G(t)=\exp \left[2\left(N_{1}-N_{2}\right) k t\right] .
$$

One can see that if $\lambda=G^{-1}$ the expression (64) coincides with (59).

Thus, the scale transform (58) of the phase space turns out to be connected with the action of the quantum amplifier. The form of this transform is determined by the structure of the Hamiltonian (62). Accordingly, the action of the amplifier at an arbitrary quantum state can be described by means of a scale transform of the phase space.

Let us find the Heisenberg and RobertsonSchrödinger uncertainty relations for the stretched states. The new method for constructing Husimi symbols for operators was proposed in $[24,25]$. It is especially effective for operator, which are polynomial of position $\hat{q}$ and momentum $\hat{p}$ operators. With such operators we will deal in this section.

Let's consider the Hamiltonian of a harmonic oscillator

$$
\hat{H}=\frac{\hbar \omega}{2}\left(\hat{q}^{2}+\hat{p}^{2}\right) \text {. }
$$

Its Husimi symbol reads

$K_{\mathrm{H}}(q, p)=q^{2}+p^{2}-1$. 
The mean value $\bar{E}$ of the energy of a state, which is characterized by Hushimi function $Q(q, p)$, has the form

$$
\begin{aligned}
& \bar{E}=\int \frac{\hbar \omega}{2}\left(q^{2}+p^{2}-1\right) Q(q, p) d q d p= \\
& =\int \frac{\hbar \omega}{2}\left(q^{2}+p^{2}\right) Q(q, p) d q d p-\frac{\hbar \omega}{2} .
\end{aligned}
$$

Let us find now the average value of the energy of a stretched state, which corresponds to a state with Husimi function $Q(q, p)$. The Husimi function of the stretched state has the form $Q_{\lambda}(q, p)=\lambda^{2} Q(\lambda q, \lambda p)$, and the average value of energy $\bar{E}_{\lambda}$ of a state with such Husimi function is determined by the formula

$\bar{E}_{\lambda}=\int K_{H}(q, p) Q_{\lambda}(q, p) d q d p=$

$=\int \frac{1}{\lambda^{2}} \frac{\hbar \omega}{2}\left((\lambda q)^{2}+(\lambda p)^{2}-1\right) Q(\lambda q, \lambda p) d(\lambda q) d(\lambda p)=$

$=\frac{1}{\lambda^{2}} \bar{E}+\frac{1-\lambda^{2}}{\lambda^{2}} \frac{\hbar \omega}{2}$.

The expression (69) is valid for all stretched states of harmonic oscillator. Since $|\lambda|^{2}<1$ it is clear that with the scale transform the energy of stretched states increases. For the Fock states the mean value of energy of corresponding stretched states can be found in explicit form. Thus, in the case of an arbitrary superposition of Fock states, we have

$$
\bar{E}_{\Sigma \lambda}=\frac{1}{\lambda^{2}} \frac{\hbar \omega}{2} \sum_{k=0}^{\infty}\left|c_{k}\right|^{2} k+\frac{1-\lambda^{2}}{\lambda^{2}} \frac{\hbar \omega}{2} .
$$

With the help of the expression for mean values of operators and using the Husimi functions, it is possible to find the form of the uncertainty relations for stretched states. We will consider the Heisenberg and Robertson-Schrödinger uncertainty relations. The relations (5), (8) are satisfied for any quantum states. Let us now establish what happens with these relations when one passes to stretched states. To do this it is necessary to calculate the quantities

$$
\begin{aligned}
& \sigma_{q q}=\left\langle\hat{q}^{2}\right\rangle-\langle\hat{q}\rangle^{2}, \sigma_{p p}=\left\langle\hat{p}^{2}\right\rangle-\langle\hat{p}\rangle^{2}, \\
& \sigma_{q p}=\frac{1}{2}\langle\hat{p} \hat{q}+\hat{q} \hat{p}\rangle-\langle\hat{q}\rangle\langle\hat{p}\rangle .
\end{aligned}
$$

With the help of Husimi function, the dispersions $\sigma_{\mathrm{qq}}, \sigma_{\mathrm{pp}}$ and the value $\sigma_{\mathrm{qp}}$ can be written in the form

$\sigma_{q q}=\int\left(q^{2}-\frac{1}{2}\right) Q(q, p) d q d p-\left(\int q Q(q, p) d q d p\right)^{2}$,

$\sigma_{p p}=\int\left(p^{2}-\frac{1}{2}\right) Q(q, p) d q d p-\left(\int p Q(q, p) d q d p\right)^{2}$,

$\sigma_{q p}=\int q p Q(q, p) d q d p-\int q Q(q, p) d q d p \int q Q(q, p) d q d p$.

For the stretched states the formulas (72) take the form

$$
\begin{aligned}
& \sigma_{q q \lambda}=\int\left(q^{2}-\frac{1}{2}\right) \lambda^{2} Q(\lambda q, \lambda p) d q d p-\left(\int q \lambda^{2} Q(\lambda q, \lambda p) d q d p\right)^{2}, \\
& \sigma_{p p \lambda}=\int\left(p^{2}-\frac{1}{2}\right) \lambda^{2} Q(\lambda q, \lambda p) d q d p-\left(\int p \lambda^{2} Q(\lambda q, \lambda p) d q d p\right)^{2}, \\
& \sigma_{q p \lambda}=\int q p \lambda^{2} Q(\lambda q, \lambda p) d q d p- \\
& -\int q \lambda^{2} Q(\lambda q, \lambda p) d q d p \int p \lambda^{2} Q(\lambda q, \lambda p) d q d p .
\end{aligned}
$$

The formulas (73) give the values $\sigma_{\mathrm{qq}}, \sigma_{\mathrm{pp}}$, $\sigma_{\mathrm{qp}}$ for the stretched states

$\sigma_{q q \lambda}=\frac{1}{\lambda^{2}} \sigma_{q q}+\frac{1-\lambda^{2}}{\lambda^{2}}, \sigma_{p p \lambda}=\frac{1}{\lambda^{2}} \sigma_{p p}+\frac{1-\lambda^{2}}{\lambda^{2}}, \sigma_{q p \lambda}=\frac{1}{\lambda^{2}} \sigma_{q p}$.

Using these expressions one can find how the Heisenberg and Robertson-Schrödinger uncertainty relations are modified upon transition to stretched states.

$$
\begin{aligned}
& \sigma_{q q \lambda} \sigma_{p p \lambda}= \\
& =\frac{1}{\lambda^{4}}\left(\sigma_{q q} \sigma_{p p}+\frac{1}{2}\left(1-\lambda^{2}\right)\left(\sigma_{q q}+\sigma_{p p}\right)+\frac{1}{4}\left(1-\lambda^{2}\right)^{2}\right) \geq \frac{1}{4 \lambda^{4}} \hbar^{2},
\end{aligned}
$$

and

$$
\begin{aligned}
& \sigma_{q q \lambda} \sigma_{p p \lambda}-\sigma_{q p \lambda}^{2}= \\
& =\frac{1}{\lambda^{4}}\left(\sigma_{q q} \sigma_{p p}-\sigma_{q p}^{2}+\frac{1}{2}\left(1-\lambda^{2}\right)\left(\sigma_{q q}+\sigma_{p p}\right)+\frac{1}{4}\left(1-\lambda^{2}\right)^{2}\right) \geq \frac{1}{4 \lambda^{4}} \hbar^{2} .
\end{aligned}
$$

We see that the right-hand sides of the uncertainty relations (75) contain a factor $\lambda^{-4}$, so for $|\lambda|<1$ its values increase and, generally speaking, can become arbitrarily large. In this respect, they are similar to correlated states. As well as in the case of correlated states, we can assume that a scale transform $(q, p) \rightarrow(\lambda q, \lambda p)$ leads to the appearance of an "effective Planck constant". For $\lambda<<1$ the effective Planck constant satisfies the inequality $\hbar_{\text {eff }} \gg \hbar$. 
However, there is a significant difference between these two types of states: the correlated states are pure states, and the stretched states are mixed states. The density matrices of these mixed states were found in [26, 27].

\section{CONCLUSION}

In this article we give an overview of the states of two types: the correlated states and the stretched states. These states can possess a great uncertainty in position and momentum, and for them the lefthand sides of Heisenberg and RobertsonSchrödinger uncertainty relations can take values significantly exceeding the minimum value. These states are obtained from the states of a harmonic oscillator with the help of nonlinear processes or external energy effects.

In the case when these conditions are realized in the atomic nucleus, the appearance of states with large values of the position and momentum dispersions can increase the probability of the tunnel effect and lead to low-energy nuclear reactions. Formally, the increase of probability of a tunnel transition can be correlated with the "increase" of the Planck constant $h$, i.e. with the ability to use the value $\hbar_{\text {eff }} \gg \hbar$ instead of $\hbar$.

It was proposed in Ref. [15-17, 33] to use this property of correlated states to describe low-energy nuclear reactions. The stretched states also have great uncertainty, but they arise in other physical processes. We hope that they also will find application in nuclear physics and in other fields where there is an increase probability of a tunnel effect.

\section{REFERENCES}

1. Vysotskii VI, Kornilova AA. Yaderny sintez. $i$ transmutatsiya izotopov $v$ biologicheskikh sistemakh [Nuclear fusion and transmutation of isotopes in biological systems]. Moscow,
MIR Publ., 2003, 302 p., ISBN 5-03-0036474 OCLC 67158435.

2. Ratis YuL. Upravlyaemy "termoyad" ili kholodny sintez? Drama idey [Controlled "fusion" or cold synthesis? Drama ideas]. http:/ / electrosad.ru>files/LENR/cold.pdf (in Russ.).

3. LENR ore not LENR? http://geektimes. ruspost/275724/ (in Russ.).

4. Zhigalov V. Russkaja mozaika LENR (nizkoenergeticheskie yadernye reaktsii) [Russian mosaic LENR]. http://electrosad. rusfiles/LENR/Zhigalov Vlad.pdf/ (in Russ.).

5. LENR - "kholodny sintez" ili "effekt Fleyshmana-Ponsa" ["Cold fusion" or "effect...]. http://genveles.livejournal. com/213196.html/ (in Russ.).

6. V zashchitu kholodnogo yadernogo sinteza [In defense of cold nuclear fusion (LENR)]. http://ss69100.livejournal.com/ (in Russ.).

7. Zel'dovich YaB, Gershtein SS. Yadernye reaktsii v kholodnom vodorode. 1. Mezonny kataliz [Nuclear reactions in cold hydrogen. I. Mesonic catalysis]. UFN, 1960, 71:581-630 (in Russ.).

8. Dodonov VV, Man'ko VI. Invariants and correlated states of nonstationary quantum systems. Proc. Lebedev Physics Institute, 1989, 183:71-181. In: Markov MA (ed.). Invariants and the Evolution of Nonstationary Quantum Systems, Commack, NY (USA); Nova Science Publishers, Inc., 1989.

9. Heisenberg W. Ztschr. Phys., 1927, 43:172-198. 10. Robertson HP. Phys. Rev., 1929, 34:163-164; Phys. Rev. A, 1930, 35(5);667.

11. Schrödinger E. About Heisenberg Uncertainty Relation (Eng. transl.) Berl. Königlich Akad. Wiss., Berlin, 1930: 296-303.

12. Dodonov VV, Dodonov AV. Transmission of correlated Gaussian packets through a delta-potential. J. Russ. Laser Res., 2014, 35(1):39-46.

13. Dodonov AV, Dodonov VV. Tunneling of slow quantum packets throw the high 
Coulomb barrier. Physics Letters A, 2014, 378:1071-1073.

14. Vorontsov YuI. Uncertainty relation and the measurement error-perturbation relation. Sov.Phys.Usp., 2005, 48(10):999-1013.

15. Vysotskii VI, Vysotskyy MV, Adamenko SV. Formation and application of correlated states in nonstationary systems at low energies of interacting particles. J. Exp. Theor. Phys., 2012, 114:243-252.

16. Vysotskii VI, Adamenko SV, Vysotskyy MV. The formation of correlated states and the increase in barrier transparency at a low particle energy in nonstationary systems with damping and fluctuations. J. Exp. Theor. Phys., 2012, 115:551-566.

17. Vysotskii VI, Vysotskyy MV. Formation of correlated states and optimization of nuclear reactions for low-energy particles at nonresonant low-frequency modulation of a potential well. J. Exp. Theor. Phys., 2015, 120:246-256.

18. Bykov VP. Basic properties of squeezed light. Sov. Phys. Usp., 1991, 34(10):910-924.

19. Mandel L, Wolf E. Optical Coherence and Quantum Optics. Cambridge University Press, 1995.

20. Yariv A. Quantum Electronics. John Sons, Inc., 1975.

21. Scully MO, Zubairy MS. Quantum Optics. Cambridge University Press, 1997.

22. Andreev VA, Davidović DM, Davidović LD, Davidović MD, Manko VI, Manko MA. A transformational property of the Husimi function and its relation to the Wigner function and symplectic tomograms. Theor. Math. Phys., 2011, 166:356-368.

23. Andreev VA, Davidovich DM, Davidovich LD, Davidovich MD. Relationships between scaling transformed Husimi functions and symplectic tomograms describing corresponding physical states. Phys. Scr., 2011, 143:01400.

24. Andreev VA, Davidović LD, Davidović Milena D, Davidović Miloš D, Manko VI,
Manko MA. Operator method for calculating Q symbols and their relation to Weyl-Wigner symbols and symplectic tomogram symbols. Theor. Math. Phys., 2014, 179:559-573.

25. Andreev VA., Davidović Milena D., Davidović LD, Davidović Miloš D and Davidović DM. Derivation of the Husimi symbols without antinormal ordering, scale transformation and uncertainty relations. Physica Scripta, 2015, 90(7):074023.

26. Vladimir A. Andreev, Dragomir M. Davidovi'c, Ljubica D. Davidovi'c, Milena D. Davidovi'c, Milo`s D. Davidovi'c, and Sergey D. Zotov, Scaling transform and stretched states in quantum mechanics. J. Russ. Laser Res., 2016, 37(5):434-439.

27. Andreev VA, Davidovi'c DM, Davidović LD, Davidović Milena D, Davidović Miloš D. The scale transformation of a phase space and stretched states of harmonic oscillator. Theor. Math. Phys., 2017, 192(1):164-184.

28. Agarwal GS, Tara K. Transformation of the nonclassical states by an optical amplifier. Phys. Rev. A, 1993, 47(4):3160-3166.

29. Agarwal GS, Chaturvedi S, Rai Amit. Amplification of NOON States, http:// arxiv.org/abs/0912.5134v1.

30. Andreev VA, Lerner PB. Supersymmetry in the Jaynes-Cummings Model. Physics Letters A, 1989, 134:507-511.

31. Andreev VA. Supersymmetry of two-level systems. J. Sov. Laser Res., 1992, 13:268-278.

32. Schleich WP. Quantum Optics in Phase Space. WILEY-VCY, 2001.

33. Chernega VN. Purity dependent uncertainty relations and possible enhancement of quantum tunneling phenomenon. J. Russ. Laser Res., 2013, 34(2):103-108; http:/ / arxiv. org/abs/1303.5238v1. 